\title{
MEMS INERTIAL POWER GENERATORS FOR BIOMEDICAL APPLICATIONS
}

\author{
P. Miao, P. D. Mitcheson, A. S. Holmes, E. M. Yeatman, T. C. Green and B. \\ H. STARK \\ Department of Electrical and Electronic Engineering, Imperial College London, \\ SW7 2BT
}

\begin{abstract}
An inertial vibration scavenging micro-generator is described which is suitable for biomedical applications. MEMS fabrication techniques have been used to construct a variable capacitor having one moving plate on which a proof mass is attached. Energy is extracted when the plates separate at constant charge. This device is non-resonant, so can operate over a wide range of excitation frequencies and amplitudes. The energy extracted per cycle, $120 \mathrm{~nJ}$, is well above previous reports. The fabrication techniques employed are described, and test data are given and compared to modeling results, showing good agreement.
\end{abstract}

\section{Introduction}

To avoid battery replacement or recharging in mobile or autonomous devices, methods for parasitic energy extraction from the environment are desirable. MEMS solutions are appropriate where space is constrained, e.g. in medical applications, and MEMS inertial generators are receiving increasing interest [1]. These devices typically use a proof mass mounted within a frame on a spring suspension, with energy extracted by an electric damping mechanism which may be electromagnetic, electrostatic or piezoelectric. Most MEMS generators reported elsewhere have been mechanically resonant structures; for this reason they are not well suited to the low frequencies of human powered applications. Previously we have introduced and analysed a new device type using nonlinear motion [2], and reported experimental results from a hybrid, meso-scale implementation [3]. Here we report on the first working device of this type fabricated by MEMS techniques.

\section{Device Structure and Operation}

Design of an energy scavenging device for biomedical applications should be optimized to the particular characteristics of such applications. A crucial characteristic is the low frequency range of body and organ motion. Acceleration 
measurements on a moving person, for example, show fundamental frequencies around $1 \mathrm{~Hz}$, but with a rich spectral content, so that optimal oscillating frequencies for attached generators can be a few $\mathrm{Hz}$ or up to a few 10's of $\mathrm{Hz}$ [4]. Secondly, a significant variation of the motion amplitude and frequency can be expected, compared to some machine mounted applications, so generators should either have high response for a wide range of input characteristics, or be dynamically tunable. This requirement may be a major impediment to the use of devices intended to operate resonantly at the frequency of the driving motion. Naturally, size should be unobtrusive for worn or implanted applications, with a volume of $1 \mathrm{~cm}^{3}$ being a reasonable limit.

Of the three electromechanical transduction methods typically used for vibration driven micro-generators, electrostatic conversion is particularly well suited to low frequency operation. Efficient energy extraction requires that the transduction mechanism presents a strong damping force to the proof mass, and this is difficult to achieve at low frequency because of the associated low velocity. For electromagnetic systems, limits on achievable magnetic gradients, and on number of coil turns in microengineered variants, result in low efficiency. Piezoelectric devices may also suffer from charge leakage at low operating frequency. For electrostatic devices, damping strength depends on the capacitance values that can be realized, but can also be increased through high voltage operation, within the limits imposed by dielectric breakdown. For microengineered applications, electrostatic implementations avoid the need for special materials (as are needed in the piezoelectric case). While their efficiency is affected by parasitic capacitances, the ohmic losses in the coils of electromagnetic versions are likely to be more severe.

Resonant operation offers significant advantage if the amplitude of the external motion is less than the maximum internal displacement of the proof mass [2]. However, this is unlikely to be the case in biomedical applications where the motions are large and the devices are small. Also, the ability to dynamically tune the mechanical resonance of the device is a very challenging requirement. For these reasons, we have pioneered a non-resonant device in which energy is maximized by allowing the proof mass only to move during that portion of the motion cycle where acceleration is maximum. 
An exploded view of the device structure is shown in Fig. 1, with the phases of operation illustrated in Fig. 2. The structure consists of a moving plate attached to a frame by a low stiffness suspension, a bottom plate containing the counter-electrode and charging studs, and a top plate with the discharge contacts. Initially the moving plate is at its lowest position, against the charging studs, and the variable capacitance is maximised. A key challenge for high performance is to maximise this initial capacitance, by minimising the gap. Application of the charging (or priming) voltage creates a holding force between the moving and fixed plate - this is the priming phase. The priming voltage is chosen to give a holding force just below the maximum inertial force (i.e. proof mass $\times$ peak acceleration), so as to maximise the output power.

When the acceleration reaches a sufficient level, the mass separates from the charging plate and is pulled across the gap at constant charge - this is the flight phase. As the capacitance drops, the voltage rises proportionally, so the stored energy $\left(\mathrm{CV}^{2} / 2\right)$ increases by the ratio of the initial to final capacitance. It then discharges into the load circuit - the conversion phase. We call the device the Coulomb force parametric generator (CFPG). To a first approximation the output power can be increased by reducing the device capacitance at discharge, but in practice this is only useful while this value remains higher than the parasitic capacitances between the moving plate and, for example, the discharge circuit. Increasing the starting capacitance has the additional advantage of reducing the required priming voltage.

The CFPG has a number of advantages over resonant structures: besides its ability to operate efficiently over a wide range of input frequencies and waveforms, it can also be optimised dynamically for operating conditions by varying the priming voltage.

\section{Fabrication}

The device was fabricated using a 3-wafer construction. The central wafer contains a silicon proof mass, forming one plate of the variable capacitor, along with a silicon frame and a polyimide suspension, metalised for electrical contact. The proof mass is about $0.12 \mathrm{~g}$, and measures $\approx 11 \times 11 \mathrm{~mm} \times 0.4 \mathrm{~mm}$ thick. It is separated from the frame by deep reactive ion etching (DRIE), through the whole 
wafer thickness, after patterning of the suspension. Polyimide is chosen to give a very low suspension stiffness, to avoid resonant effects.

The bottom wafer is glass, to minimise the parasitic capacitance. It includes the fixed electrode of the variable capacitor itself, and the charging studs and spacers for the moving plate and middle wafer, the studs being deposited by electroplating. These set the minimum gap at about $6 \mathrm{um}$, giving a theoretical starting capacitance of $\approx 180 \mathrm{pF}$. The measured (static) starting capacitance was $\approx$ $150 \mathrm{pF}$, the difference being attributed to wafer bow. The top wafer is also glass, and has studs for discharge. Spacer studs 300 um thick, fabricated from SU8 polymer on both top and bottom wafers, set the layer separation, and thus the proof mass travel distance. The minimum (discharge position) capacitance was measured at 5.5 pF. Fig. 3 shows the completed device, while Fig. 4 shows a detail of the suspension on one edge.

We have investigated possible switched-mode circuits for converting the high voltage output pulses from the generator into a useable low-voltage form. This application places unique demands on the semiconductor devices because of the linked requirements of high blocking voltage and very low parasitic capacitance and leakage currents. Nevertheless, we have been able to design suitable devices in SOI technology using standard design rules; this work is described in detail in [5].

\section{Device Testing}

The device was tested on a low frequency shaker platform, for frequencies in the range $10-100 \mathrm{~Hz}$. Reproducible results were not obtained at lower frequencies; this is partly because the holding force is not yet sufficient to optimize performance at these frequencies, and also because of limits on the maximum shaker displacement amplitude. Motion was monitored using a linear displacement transducer or an accelerometer at lower and higher frequencies respectively. Although monitoring of the moving plate voltage during operation is not required in a working device, it was carried out here for diagnostic purposes. This necessitated development of an instrumentation amplifier with input resistance above $10^{12} \Omega$, and input capacitance below $1 \mathrm{pF}$, as described in [3]. The measured moving plate voltage was compared to an electromechanical simulation developed in SPICE (Fig. 5); a close correspondence is observed. 
As the pre-charge voltage (and thus the holding force) is increased, the release point should occur later in the cycle. Experiments were carried out to verify this, and again the behaviour matches well the simulations (Fig. 6).

\section{Analysis}

Depending on operating frequency and amplitude, output voltages of up to $220 \mathrm{~V}$ were obtained, corresponding to a net generated power of $120 \mathrm{~nJ}$ per cycle. This is well above previously reported values for MEMS electrostatic generators [6], which are typically a few $\mathrm{nJ} /$ cycle or less.

However, the power obtained remains significantly below theoretically achievable values. We believe an important limitation in this case is the motion of the proof mass in unwanted degrees of freedom; in particular, tilting motion. This reduces the capacitance ratio, by decreasing the charging capacitance if the moving plate is not parallel to the fixed plate and does not contact all the charging studs, and by increasing the discharge capacitance. The dynamic starting capacitance, in particular, was found to deviate significantly from the static value, at only $\approx 50 \mathrm{pF}$. This corresponds to a much greater effective minimum plate separation (assuming parallel plates) of about $21 \mu \mathrm{m}$. In fact, the experimental results provide further evidence that the effective capacitance ratio during operation is below the design value. The maximum inertial force $F_{\mathrm{i}}$ is $\operatorname{ma} a_{\max }$, and with $\mathrm{m}=0.12 \mathrm{~g}$ and a maximum acceleration $\mathrm{a}_{\max }$ of $\approx 40 \mathrm{~m} / \mathrm{s}^{2}$ (Fig. 5), $\mathrm{F}_{\mathrm{i}}$ does not go above $\approx 5 \mathrm{mN}$. The electrostatic holding force is given by [2]:

$$
\mathrm{F}_{\mathrm{e}}=\frac{1}{2} \mathrm{~V}^{2} \frac{\mathrm{C}}{\mathrm{d}_{\min }}
$$

Taking the design values of $\mathrm{C}=180 \mathrm{pF}$ and minimum plate separation $\mathrm{d}_{\min }=6$ $\mu \mathrm{m}$, and the applied priming voltage of $30 \mathrm{~V}$ (Fig. 5), we get $\mathrm{F}_{\mathrm{e}}=13.5 \mathrm{mN}$, suggesting that the inertial force is never sufficiently strong to release the mass. With the measured effective (dynamic) capacitance of $50 \mathrm{pF}$ and an increased minimum plate separation, $F_{e}$ falls below the maximum inertial force as expected.

Another deviation that can be seen in the experimental results is the late landing of the moving plate on the charging studs, and the slower than predicted transit towards the discharging studs. Viscous air damping provides a likely explanation for these differences. The trapped air can be modelled as a linear spring and a viscous damper in parallel, and for a relatively slow moving (low 
frequency) system, the latter can be shown to dominate [7]. In this case the damping force can be approximated by:

$$
\mathrm{F}_{\mathrm{d}}=\frac{0.4 \mu \mathrm{A}^{2}}{\mathrm{~d}^{3}} \mathrm{v}
$$

with $\mu$ the air viscosity, A the plate area, $d$ the separation and $v$ the velocity. Taking the viscosity as $15 \times 10^{-6} \mathrm{~kg} / \mathrm{ms}$ [7], and $\mathrm{v} \cong 0.1 \mathrm{~m} / \mathrm{s}$ (Fig. 5), the damping force exceeds both $F_{i}$ and $F_{e}$ for plate separations below about $100 \mu \mathrm{m}$. Although this simplified model becomes less accurate for the smaller separations, it is never-the-less likely that air damping significantly influences the behaviour, both in slowing the transit and in encouraging tilting of the moving plate.

Future designs will address these issues by better restraining motion in unwanted axes, through a modified suspension, and reducing air damping through perforation of the plates (operation in vacuum is also a possibility). Even if these improvements are made, the starting capacitance should be increased from its current design value, in order to reduce the priming voltage at which the optimum holding force is obtained and to reduce the impact of parasitics on the achievable capacitance ratio.

Let us now examine the ultimate realisable power density. In [2] we showed that the theoretically achievable power from vibration powered generators is given by:

$$
\mathrm{P} \approx \frac{1}{2} \mathrm{Y}_{\mathrm{o}} \mathrm{Z}_{1} \omega^{3} \mathrm{~m}
$$

for excitation amplitude $Y_{0}$, frequency $\omega$, internal displacement $Z_{1}$ and proof mass m. This holds for the CFPG or for resonant devices operating at resonance. Let us now assume for the generator shape a flat square of area $\mathrm{a} \times \mathrm{a}$, and thickness $\alpha \mathrm{a}$, with $\alpha$ an aspect ratio $<1$. The optimal mass will occupy approximately half the volume, leaving an internal displacement amplitude $\alpha \mathrm{a} / 4$ for vertical motion. Then for a proof mass density $\rho$, and substituting for $\mathrm{Y}_{\mathrm{o}} \omega^{2}$ the maximum external acceleration $\mathrm{A}_{0}$, we have:

$$
\mathrm{P} \approx \frac{1}{160} \mathrm{~A}_{\mathrm{o}} \omega \rho \alpha \mathrm{a}^{4}
$$

A peak acceleration of about $1 \mathrm{~g}\left(10 \mathrm{~m} / \mathrm{s}^{2}\right)$ is typical of body motion [4]. Then, taking an aspect ratio $\alpha=0.2$, and assuming for $\rho$ a silicon proof mass, we obtain: 


$$
\mathrm{P} \approx 180 f a^{4} \mathrm{~W} / \mathrm{m}^{4} \mathrm{~Hz}
$$

with $f=\omega / 2 \pi$. This is plotted in Fig. 7 for two values of device size a. For our current device having a $=11 \mathrm{~mm}$, for operation at $30 \mathrm{~Hz}$, about $80 \mu \mathrm{W}$ should be achievable, or $2.6 \mu \mathrm{J} /$ cycle. Using gold as the proof mass would give an increase of $10 \times$ in these figures.

Because achieving maximum power requires setting the priming voltage (and thus the holding force) to an optimal value for any given source acceleration, for a variable source such as the human body this optimisation should be carried out dynamically. Fig. 8 gives a block diagram of a possible power supply module for such a case. Here the switching is assumed to be electrical rather than mechanical as in the current prototype, and both the charging voltage and the charging and discharge timings are controlled dynamically.

\section{Conclusions}

In conclusion, parametric generators fabricated by MEMS techniques have been demonstrated, with performance closely agreeing with device models, and high output energy per cycle has been obtained. These devices are promising for low frequency applications such as wearable or implantable devices. The key challenges remaining for the technology are to improve the resistance to motion in unwanted modes (particularly tilting), to further reduce parasitics, and to integrate the devices with the associated power electronics.

\section{References}

[1] S. Roundy, P.K. Wright and J.S. Rabaey, Energy Scavenging for Wireless Sensor Networks, Boston: Kluwer, 2004.

[2] P.D. Mitcheson, T. C. Green, E. M. Yeatman and A. S. Holmes, J. MEMS 13, 429-440 (2004).

[3] Mitcheson P.D., Miao P., Stark B.H., Yeatman E.M., Holmes A.S., Green T.C., Sensors and Actuators 115, 523-529 (2004).

[4] T. von Buren, P. D. Mitcheson, T. C. Green, E. M. Yeatman, A. S. Holmes, G. Troster, "Optimization of Inertial Micropower Generators for Human Walking Motion", IEEE Sensors Journal, accepted.

[5] B.H. Stark et al., Proc. $35^{\text {th }}$ Ann. IEEE Power Electronics Specialist Conference (PESC '04), 4156-4162 (2004).

[6] R. Amirtharajah \& A.P. Chandrakasan, IEEE J. Solid State Circuits 33, 687-695 (1998).

[7] M. Andrews, I. Harris and G. Turner, Sensors and Actuators 36, 79-87 (1993). 


\section{Figure Captions}

Fig. 1: Exploded view of the generator construction.

Fig. 2 : Generator operation phases: Prime, Wait, Flight and Conversion.

Fig. 3 : Prototype generator.

Fig. 4: Detail of middle wafer showing suspension strips and anchor points.

Fig. 5 : Moving plate voltage and frame acceleration.

Fig. 6 : Acceleration at release point vs. pre-charge voltage.

Fig. 7 : Maximum output power vs. frequency, for dimensions as indicated.

Fig. 8 : Block diagram for vibration-powered autonomous sensor supply module. 


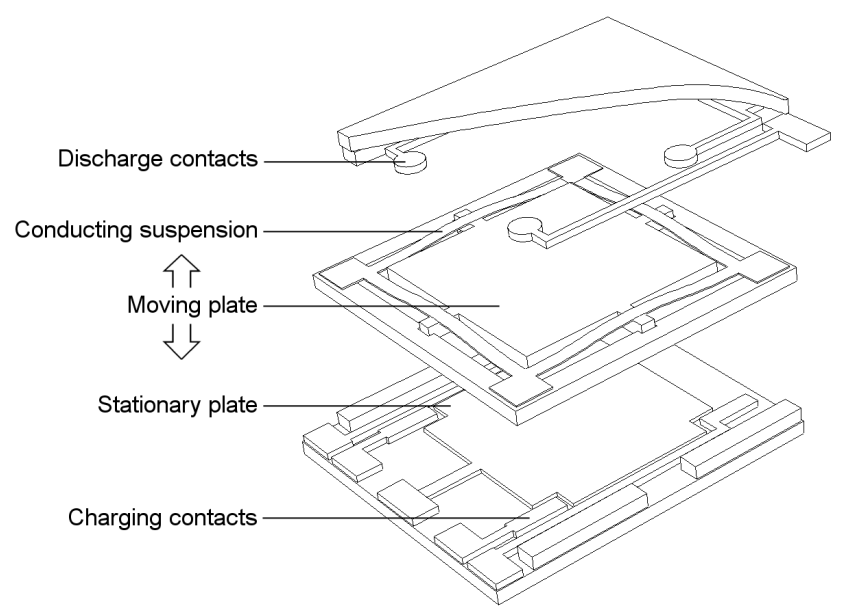

Fig. 1: Exploded view of the generator construction.

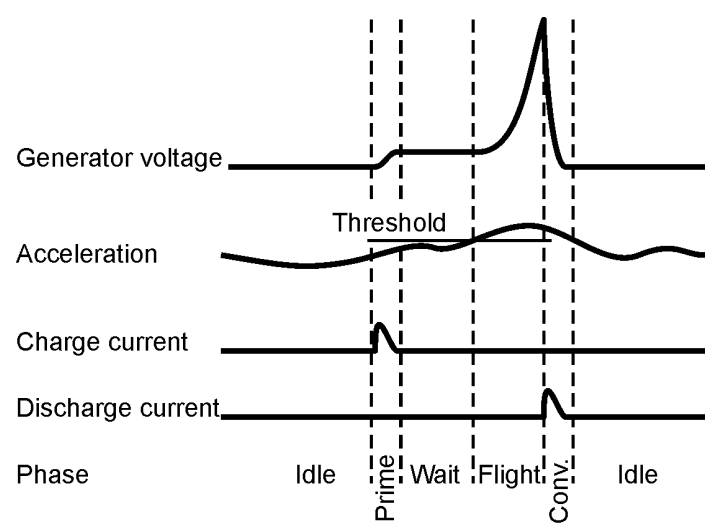

Fig. 2 : Generator operation phases: Prime, Wait, Flight and Conversion.

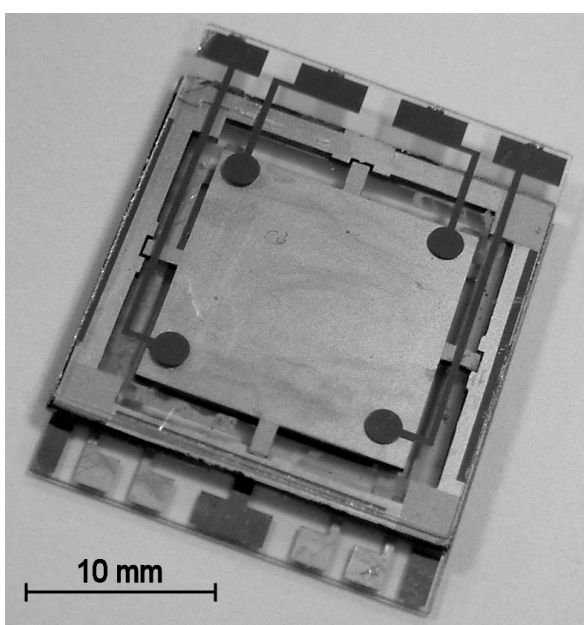

Fig. 3 : Prototype generator. 


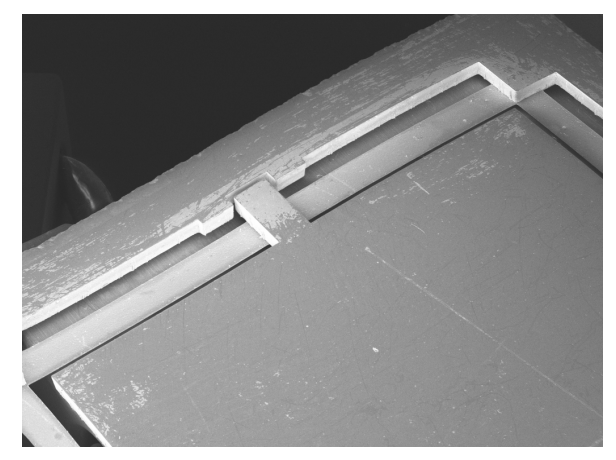

Fig. 4: Detail of middle wafer showing suspension strips and anchor points.
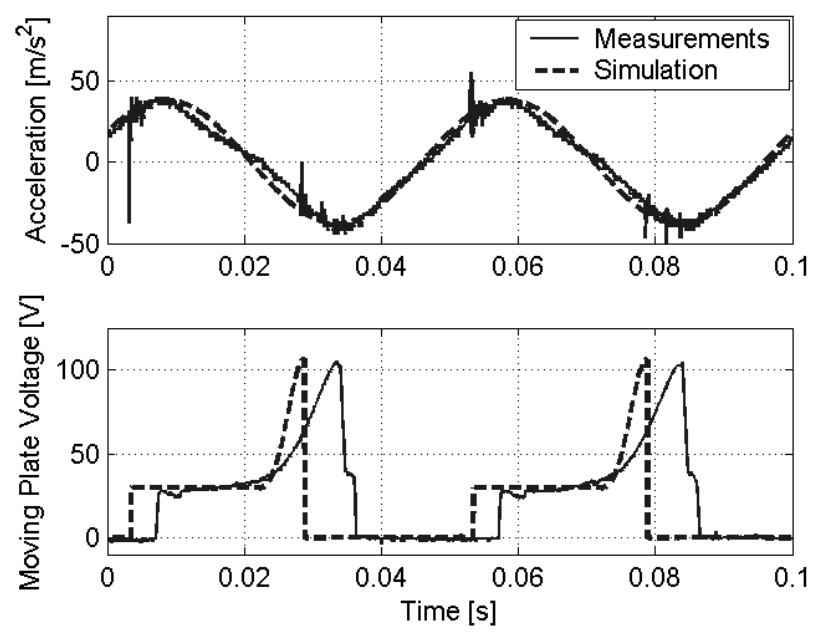

Fig. 5 : Moving plate voltage and frame acceleration.

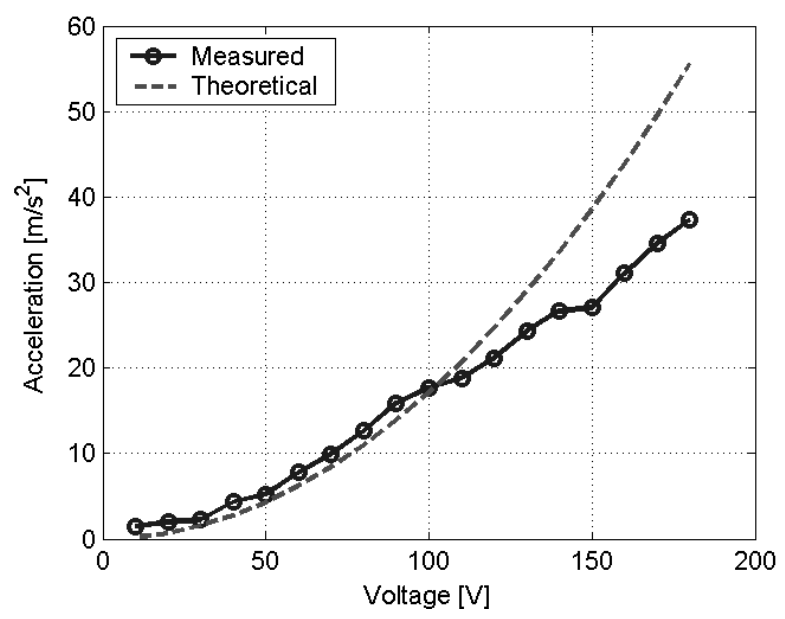

Fig. 6 : Acceleration at release point vs. pre-charge voltage. 


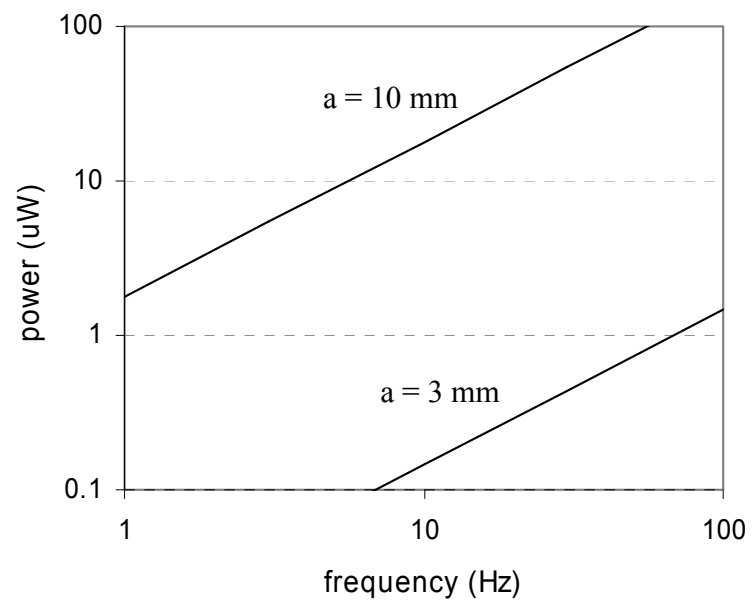

Fig. 7 : Maximum output power vs. frequency, for dimensions as indicated.

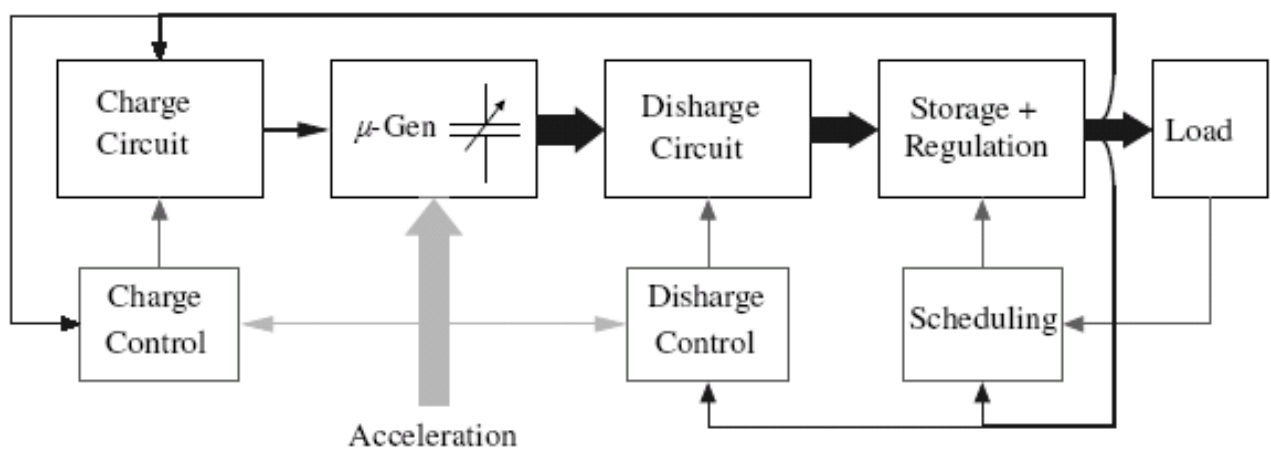

Electrical Energy Control Signal $\square$ Kinetic Energy

Fig. 8 : Block diagram for vibration-powered autonomous sensor supply module. 